\title{
Author Correction: Highly specific multiplexed RNA imaging in tissues with split-FISH
}

Jolene Jie Lin Goh, Nigel Chou, Wan Yi Seow, Norbert Ha D, Chung Pui Paul Cheng, Yun-Ching Chang, Ziqing Winston Zhao and Kok Hao Chen (D)

Correction to: Nature Methods https://doi.org/10.1038/s41592-020-0858-0, published online 15 June 2020.

In the version of Supplementary Table 3 originally posted for this article, an extra $\mathrm{G}$ nucleotide was inserted in error into all of the bridge sequences. The error has been corrected online.

Published online: 19 November 2020

https://doi.org/10.1038/s41592-020-01019-w

() The Author(s), under exclusive licence to Springer Nature America, Inc. 2020 